\title{
Research on the Post Evaluation for Thermal Power Construction Project Based on Entropy Weight Fuzzy Comprehensive Evaluation
}

\author{
Jianna Zhao \\ Department of Economics and Management \\ North China Electric Power University \\ Baoding, China \\ zhjnzhf@163.com
}

\begin{abstract}
In order to improve decision-making and investment returns on a construction project, in this paper, entropy weight fuzzy model is employed to make post evaluation for thermal power construction project in combining with success evaluation theory. After fully consider the features of thermal power construction project, this paper establishes the indicator system of post evaluation from four aspects: economy, process, effect and sustainability. With entropy method to calculate the weight, it is concluded that, through a specific example, the evaluation result is good by fuzzy comprehensive estimation.
\end{abstract}

Keywords-thermal power construction project; post evaluation; entropy weight fuzzy comprehensive evaluation

\section{INTRODUCTION}

Post-project Evaluation refers to a kind of technical and economic activity which makes systematic and objective analysis and summary to the purpose, execution, efficiency, effect and influence of the project (or program) when the project has been completed and run for a period of time. Through check and summarize to the project activities, it will make sure whether the project reaches its anticipated target and, especially, if the main benefit goal be achieved. Besides, it's necessary to find out the cause(s) of failures through the analysis and evaluation and sum up experiences and learn from the failures. Furthermore, the information produced during the process of analysis and evaluation, as a basis for future project decisions, would be timely feedback. The post evaluation for thermal power construction project is helpful to improve executors' management and investors' decision-making level, which make them to accurately grasp the direction of investment and improve investment benefits. Therefore, this is realistic and necessary to research the post evaluation for thermal power construction project.

\author{
Yuanyuan Zhao
}

Department of Economics and Management

North China Electric Power University

Baoding, China

1508151222@qq.com

\section{GENERAL METHODS FOR POST EVALUATION ON} CONSTRUCTION PROJECT

On the basis of following the general principles of evaluation, general methods of post evaluation to construction project are: logic frame method, comparative method, the principle and method of statistics and prediction, decision tree, success degree method and index system of comprehensive evaluation method. This paper focuses on the success degree method and the index system of comprehensive evaluation method.

\section{A. Success Method}

Within general methods of post evaluation, the success degree method is a very commonly used one. With the system criterion or the objective judgment table, it depends on experts or expert group's experience to evaluate the overall success degree according to the executive condition of all aspects of a project. The success degree method evaluates projects in a comprehensive and systematic way, which takes the realization degree of an item target and the evaluation conclusion of economic benefit analysis as the core.

\section{B. Index System of Comprehensive Evaluation Method}

Index system of comprehensive evaluation method is a systematic evaluation method which combines quantitative analysis and qualitative analysis together. It has the basic application framework as follows: build the evaluation index system; determine the weight of each evaluation index; establish the comprehensive score type; sort with the comprehensive score of the project. This paper focuses on the fuzzy comprehensive evaluation method combined with using entropy to calculate the weight.

\section{ESTABLISH THE EVALUATION INDEXES OF \\ THERMAL POWER CONSTRUCTION PROJECT}

Based on the scientific, systematic, objective and comparable principle of index selection, this paper builds an index system of post evaluation from four key areas: economy, process, impact, sustainable development, see chart 3-1: 
TABLE I. POST EVALUATION INDEX TABLE OF THERMAL POWER CONSTRUCTION PROJECT

\begin{tabular}{|c|c|c|c|}
\hline & First class indexes & Second class indexes & Third class indexes \\
\hline \multirow{22}{*}{ 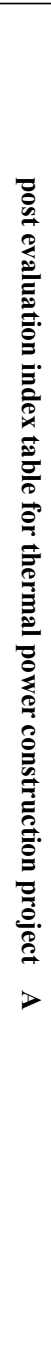 } & \multirow{5}{*}{$\begin{array}{c}\text { Economic } \\
\text { post evaluation } \\
\mathrm{B}_{1}\end{array}$} & \multirow{3}{*}{ Financial evaluation $C_{1}$} & internal rate of return $D_{1}$ \\
\hline & & & earning power of real assets $D_{2}$ \\
\hline & & & asset-liability ratio $D_{3}$ \\
\hline & & \multirow{2}{*}{ National economic evaluation $\mathrm{C}_{2}$} & $\begin{array}{l}\text { National economic internal rate of return } \\
D_{4}\end{array}$ \\
\hline & & & net present value of foreign exchange $\mathrm{D}_{5}$ \\
\hline & \multirow{11}{*}{$\begin{array}{c}\text { process } \\
\text { post evaluation } \\
\mathrm{B}_{2}\end{array}$} & \multirow{3}{*}{ Planning stage evaluation $\mathrm{C}_{3}$} & $\begin{array}{l}\text { Decision-making procedures and methods } \\
\mathrm{D}_{6}\end{array}$ \\
\hline & & & Feasibility study $\mathrm{D}_{7}$ \\
\hline & & & Risk Assessment $\mathrm{D}_{8}$ \\
\hline & & \multirow{6}{*}{ implementation stage evaluation $\mathrm{C}_{4}$} & Design work evaluation $\mathrm{D}_{9}$ \\
\hline & & & Construction work evaluation $\mathrm{D}_{10}$ \\
\hline & & & supervision evaluation $\mathrm{D}_{11}$ \\
\hline & & & Complete and start evaluation $\mathrm{D}_{12}$ \\
\hline & & & $\begin{array}{c}\text { progress, quality and safety evaluation } \\
D_{13}\end{array}$ \\
\hline & & & $\begin{array}{c}\text { Evaluation of construction management } \\
D_{14}\end{array}$ \\
\hline & & \multirow{2}{*}{ Operation stage evaluation $\mathrm{C}_{5}$} & $\begin{array}{l}\text { Efficiency of Production and operation } \\
D_{15}\end{array}$ \\
\hline & & & organization and management $\mathrm{D}_{16}$ \\
\hline & \multirow{5}{*}{$\begin{array}{c}\text { impact } \\
\text { assessment } \\
\mathrm{B}_{3}\end{array}$} & \multirow{2}{*}{ economic impact assessment $\mathrm{C}_{6}$} & Technical progress $\mathrm{D}_{17}$ \\
\hline & & & provincial economy $\mathrm{D}_{18}$ \\
\hline & & \multirow{2}{*}{ Environmental impact assessment $\mathrm{C}_{7}$} & pollution control $\mathrm{D}_{19}$ \\
\hline & & & $\begin{array}{l}\text { Capacity for Environment Management } \\
\qquad D_{20}\end{array}$ \\
\hline & & Social impact assessment $\mathrm{C}_{8}$ & \\
\hline & $\begin{array}{c}\text { sustainable development } \\
\mathrm{B}_{4}\end{array}$ & & \\
\hline
\end{tabular}

\section{EMPIRICAL ANALYSIS}

To alleviate the power shortage situation of power grid in southern Hebei, Hebei electric power company, Hebei construction investment company,Germany Siemens company and Hamburg power company jointly established the Hebei G Power Generation Co.Ltd and invested to construct $\mathrm{G}$ power plant. The power plant's planning capacity is $2400 \mathrm{MW}$, and $2 * 660 \mathrm{MW}$ coal-fired generating units would be built in the first phase project.

\section{A. Set up Factor Set and Fuzzy Appraisal Set}

First of all, the factor set of thermal power construction project can be set as $U=\left(u_{1}, u_{2}, \ldots u_{n}\right)$, according to the characteristics of the project and fuzzy appraisal set can be set as $C=\left(C_{1}, C_{2}, \ldots, C_{m}\right)=$ (excellent, good, medium, poor), in which, $U_{i}$ is a single index and $C_{j}$ is the evaluation level to $U_{i}$.

\section{B. Establish Fuzzy Evaluation Matrix}

According to success method, ten experts judge the qualitative indexes and score statistics, the results are shown in table 5-1:

TABLE II. RESULT OF INDEX FOR POST PROJECT EVALUATION

\begin{tabular}{|c|c|c|c|c|c|}
\hline & & excellent & good & medium & poor \\
\hline \multirow{3}{*}{$R_{C 1}$} & internal rate of return & 0 & 2 & 6 & 2 \\
\cline { 2 - 6 } & earning power of real assets & 0 & 4 & 6 & 0 \\
\cline { 2 - 6 } & asset-liability ratio & 0 & 3 & 7 & 0 \\
\hline \multirow{3}{*}{$R_{C 2}$} & $\begin{array}{c}\text { National economic internal rate } \\
\text { of return }\end{array}$ & 0 & 3 & 7 & 0 \\
\cline { 2 - 6 } & $\begin{array}{c}\text { net present value of foreign } \\
\text { exchange }\end{array}$ & 0 & 2 & 8 & 0 \\
\hline
\end{tabular}




\begin{tabular}{|c|c|c|c|c|c|}
\hline \multirow{3}{*}{$R_{C 3}$} & $\begin{array}{l}\text { Decision-making procedures } \\
\text { and methods }\end{array}$ & 0 & 6 & 4 & 0 \\
\hline & Feasibility study & 0 & 3 & 2 & 5 \\
\hline & Risk Assessment & 0 & 3 & 3 & 4 \\
\hline \multirow{6}{*}{$R_{C 4}$} & Design work evaluation & 3 & 7 & 0 & 0 \\
\hline & Construction work evaluation & 0 & 4 & 6 & 0 \\
\hline & supervision evaluation & 5 & 2 & 0 & 0 \\
\hline & Complete and start evaluation & 7 & 3 & 0 & 0 \\
\hline & $\begin{array}{c}\text { progress, quality and safety } \\
\text { evaluation }\end{array}$ & 6 & 3 & 1 & 0 \\
\hline & $\begin{array}{c}\text { Evaluation of construction } \\
\text { management }\end{array}$ & 4 & 4 & 2 & 0 \\
\hline \multirow{2}{*}{$R_{C 5}$} & $\begin{array}{c}\text { Efficiency of Production and } \\
\text { operation }\end{array}$ & 4 & 4 & 2 & 0 \\
\hline & organization and management & 2 & 8 & 0 & 0 \\
\hline \multirow{2}{*}{$R_{C 6}$} & Technical progress & 6 & 4 & 0 & 0 \\
\hline & provincial economy & 5 & 5 & 0 & 0 \\
\hline \multirow{2}{*}{$R_{C 7}$} & pollution control & 0 & 2 & 8 & 0 \\
\hline & $\begin{array}{c}\text { Capacity for Environment } \\
\text { Management }\end{array}$ & 0 & 1 & 7 & 2 \\
\hline \multirow[t]{2}{*}{$R_{C 8}$} & Social impact assessment & 1 & 6 & 3 & 0 \\
\hline & Sustainable development & 3 & 5 & 2 & 0 \\
\hline
\end{tabular}

Through the fuzzy statistical method, fuzzy process the quantitative indexes in the judge table and get the third level of evaluation matrix as follows:

$$
\begin{aligned}
& R_{C 1}=\left[\begin{array}{llll}
0 & 0.2 & 0.6 & 0.2 \\
0 & 0.4 & 0.6 & 0 \\
0 & 0.3 & 0.7 & 0
\end{array}\right] \\
& R_{C 2}=\left[\begin{array}{llll}
0 & 0.3 & 0.7 & 0 \\
0 & 0.2 & 0.8 & 0
\end{array}\right] \\
& R_{C 3}=\left[\begin{array}{lllc}
0 & 0.6 & 0.4 & 0 \\
0 & 0.3 & 0.2 & 0.5 \\
0 & 0.3 & 0.3 & 0.4
\end{array}\right] \\
& R_{C 4}=\left[\begin{array}{llll}
0.3 & 0.7 & 0 & 0 \\
0 & 0.4 & 0.6 & 0 \\
0.3 & 0.5 & 0.2 & 0 \\
0.7 & 0.3 & 0 & 0 \\
0.6 & 0.3 & 0.1 & 0 \\
0.4 & 0.4 & 0.2 & 0
\end{array}\right] \\
& R_{C 5}=\left[\begin{array}{cccc}
0.4 & 0.4 & 0.2 & 0 \\
0.2 & 0.8 & 0 & 0
\end{array}\right] \\
& R_{C 6}=\left[\begin{array}{llll}
0.6 & 0.4 & 0 & 0 \\
0.5 & 0.5 & 0 & 0
\end{array}\right] \\
& R_{C 7}=\left[\begin{array}{lllc}
0 & 0.2 & 0.8 & 0 \\
0 & 0.1 & 0.7 & 0.2
\end{array}\right] \\
& R_{C 8}=\left[\begin{array}{llll}
0.1 & 0.6 & 0.3 & 0
\end{array}\right]
\end{aligned}
$$

C. Calculate the Weight with Entropy Method

$H_{D 1}=-\frac{1}{\log 4}(0.2 \times \log 0.2 \times 2+0.6 \times \log 0.6)=0.453$

$H_{D 2}=-\frac{1}{\log 4}(0.4 \times \log 0.4+0.6 \times \log 0.6)=-0.511$

$H_{D 3}=-\frac{1}{\log 4}(0.3 \times \log 0.3+0.7 \times \log 0.7)=0.44$

So $H_{C 1}=\left(H_{D 1}, H_{D 2}, H_{D 3}\right)=(0.453,-0.511,0.44)$
According to the formula $V=1-H$, it draws $V_{C 1}=\left(V_{D 1}, V_{D 2}, V_{D 3}\right)=(0.547,1.511,0.56)$ 、

According to the formula $W_{i j}=V_{i j} / \sum_{j=1}^{m} V_{i j}$, it draws $W_{C 1}=\left(W_{D 1}, W_{D 2}, W_{D 3}\right)=(0.209,0.577,0.214)$

Similarly:

$$
\begin{aligned}
H_{C 2} & =\left(H_{D 4}, H_{D 5}\right)=(0.44,0.36) \\
V_{C 2} & =\left(V_{D 4}, V_{D 5}\right)=(0.56,0.64) \\
W_{C 2} & =\left(W_{D 4}, W_{D 5}\right)=(0.467,0.534) \\
H_{C 3} & =\left(H_{D 6}, H_{D 7}, H_{D 8}\right)=(0.485,0.743,0.785) \\
V_{C 3} & =\left(V_{D 6}, V_{D 7}, V_{D 8}\right)=(0.515,0.257,0.215) \\
W_{C 3} & =\left(W_{D 6}, W_{D 7}, W_{D 8}\right)=(0.522,0.26,0.218) \\
H_{C 4} & =\left(H_{D 9}, H_{D 10}, H_{D 11}, H_{D 12}, H_{D 13}, H_{D 14}\right) \\
& =(0.44, .0 .485,0.743,0.44,0.648,0.76) \\
V_{C 4} & =\left(V_{D 9}, V_{D 10}, V_{D 11}, V_{D 12}, V_{D 13}, V_{D 14}\right) \\
& =(0.56,0.515,0.257,0.56,0.352,0.24) \\
W_{C 4} & =\left(W_{D 9}, W_{D 10}, W_{D 11}, W_{D 12}, W_{D 13}, W_{D 14}\right) \\
& =(0.225,0.207,0.103 .0 .225,0.142,0.097) \\
H_{C 5} & =\left(H_{D 15}, H_{16}\right)=(0.76,0.36) \\
V_{C 5} & =\left(V_{D 15}, V_{D 16}\right)=(0.24,0.64) \\
W_{C 5} & =\left(W_{D 15}, W_{D 16}\right)=(0.273,0.727) \\
H_{C 6} & =\left(H_{D 17}, H_{D 18}\right)=(0.485,0.5)
\end{aligned}
$$


$V_{C 6}=\left(V_{D 17}, V_{D 18}\right)=(0.515,0.5)$

$W_{C 6}=\left(W_{D 17}, W_{D 18}\right)=(0.507,0.493)$

$H_{C 7}=\left(H_{D 19}, H_{D 20}\right)=(0.36,0.578)$

$V_{C 7}=\left(V_{D 19}, V_{D 20}\right)=(0.64,0.422)$

$W_{C 7}=\left(W_{D 19}, W_{D 20}\right)=(0.603,0.397)$

$H_{C 8}=0.648 ; V_{C 8}=0.352$

Using the third class indicators' data which have been draw to calculate the weights of second class indicators. By using the formula, it can be obtained:

$V_{C 1}=\sum_{j=1}^{3} V_{D j}=2.618 ; \quad V_{C 2}=\sum_{j=4}^{5} V_{D j}=1.2 ;$

$V_{B 1}=V_{C 1}+V_{C 2}=3.818$;

Similarly: $V_{B 2}=V_{C 3}+V_{C 4}+V_{C 5}=4.351$;

$V_{B 3}=V_{C 6}+V_{C 7}+V_{C 8}=2.429$

So it comes to the weight of each second class index:

$W_{C 1}=0.686, W_{C 2}=0.314, W_{C 3}=0.227, W_{C 4}=0.57$

$W_{C 5}=0.202, W_{C 6}=0.418, W_{C 7}=0.438, W_{C 8}=0.145$

Using the second class indicators' data which have been draw to calculate the weights of first class indicators. With the formula, it obtains:

$H_{B 4}=0.743 \quad V_{B 4}=0.257$

Through the known results:

$V_{B 1}=3.818 ; V_{B 2}=4.351 ; V_{B 3}=2.429$

it further gets: $\sum_{j=1}^{4} V_{B j}=V_{B 1}+V_{B 2}+V_{B 3}+V_{B 4}=10.855$,

so $W_{B 1}=0.352, W_{B 2}=0.4, W_{B 3}=0.224, W_{B 4}=0.024$

The calculation results of entropy weight is

$W_{A}=(0.352,0.4,0.224,0.024)$

\section{Fuzzy Comprehensive Evaluation}

1) fuzzy evaluation of third class

$C_{1}=W_{C 1} \cdot R_{C 1}=(0,0.337,0.621,0.041)$

$C_{2}=W_{C 2} \cdot R_{C 2}=(0,0.247,0.754,0)$

$C_{3}=W_{C 3} \cdot R_{C 3}=(0,0.457,0.326,0.217)$

$C_{4}=W_{C 4} \cdot R_{C 4}=(0.38,0.44,0.178,0)$

$C_{5}=W_{C 5} \cdot R_{C 5}=(0.255,0.69,0.055,0)$

$C_{6}=W_{C 6} \cdot R_{C 6}=(0.55,0.45,0,0)$

$C_{7}=W_{\mathrm{C} 7} \cdot R_{C 7}=(0,0.16,0.76,0.079)$

$C_{8}=W_{\mathrm{C} 8} \cdot R_{C 8}=(0.015,0.087,0.044,0)$
It obtains the second class evaluation matrix:

$$
\begin{aligned}
R_{B 1} & =\left[\begin{array}{llll}
0 & 0.377 & 0.621 & 0.041 \\
0 & 0.247 & 0.754 & 0
\end{array}\right] \\
R_{B 2} & =\left[\begin{array}{cccc}
0 & 0.457 & 0.326 & 0.217 \\
0.38 & 0.44 & 0.178 & 0 \\
0.255 & 0.69 & 0.055 & 0
\end{array}\right] \\
R_{B 3} & =\left[\begin{array}{cccc}
0.55 & 0.45 & 0 & 0 \\
0 & 0.16 & 0.76 & 0.079 \\
0.015 & 0.087 & 0.044 & 0
\end{array}\right]
\end{aligned}
$$

2) fuzzy evaluation of second class

$$
\begin{aligned}
& B_{1}=W_{B 1} \cdot R_{B 1}=\left(\begin{array}{llll}
0, & 0.337 & 0.663 & 0.028
\end{array}\right) \\
& B_{2}=W_{B 2} \cdot R_{B 2}=\left(\begin{array}{llll}
0.268, & 0.494 & 0.187 & 0.049
\end{array}\right) \\
& B_{3}=W_{B 3} \cdot R_{B 3}=\left(\begin{array}{llll}
0.232, & 0.27 & 0.34 & 0.035
\end{array}\right)
\end{aligned}
$$

It obtains the first class evaluation matrix by above formulas :

$R_{A}=\left[\begin{array}{cccc}0 & 0.337 & 0.663 & 0.028 \\ 0.268 & 0.494 & 0.187 & 0.094 \\ 0.232 & 0.27 & 0.34 & 0.035 \\ 0.3 & 0.5 & 0.2 & 0\end{array}\right]$

3) fuzzy evaluation of first class

$A=W_{A} \cdot R_{A}=(0.166,0.389,0.389,0.055)$

\section{E. Result}

It reaches a conclusion of entropy weight fuzzy comprehensive evaluation by the outcome above. In accordance with the principle of maximum membership degree, the result of entropy weight fuzzy comprehensive evaluation for the first phase of $\mathrm{G}$ power plant is good.

\section{REFERENCES}

[1] Danech-pajouh M, Sauvadet V. A statistical consistency method for evaluating the output from traffic simulation and forecasting models[C]. 2003.

[2] Eid A, Farag A. A Unified Framework for Performance Evaluation of 3-D Reconstruction Techniques[C]. 2004.

[3] Eldukair Z.A, Ayyub B.Multi-attribute fuzzy decisions in construction strategies[J]. Fuzzy sets and systems, 1992, 42(2): 155-165

[4] Wang MJJ.Fuzzy set evaluation of inspection performance [J].International journal of man-machine studies, 1991, 35(4): 587-596

[5] Christer. Carllsson, Robert, Fuller. A fuzzy approach to real option valuation [J]. Fuzzy sets and Systems,2003, 139 (4): 297-313

[6] Castro R M, Ferreira L A. A comparison between chronological and probabilistic methods to estimate wind power capacity credit[J]. Power Systems, IEEE Transactions on Power Systems. 2001, 16(4): 904-909.

[7] Xuewen T,Sifeng L, Zhigeng F, et al. Research of Rrogram Process Time Post-evaluation Grey Critical Path Algorithm Method Based on Grey Data Advantage Relationship[C]. 2006.

[8 ] Liang G S.Theory and methodology: Fuzzy MCDM based on ideal and antidotal concepts[J]. European Journal of Operational Research, 2008, 20(2): 682-691 